OPEN ACCESS

Edited by:

Malgorzata Pac

Children's Memorial Health Institute, Poland

Reviewed by:

Saul Oswaldo Lugo Reyes,

National Institute of Pediatrics, Mexico

Anders Fasth,

University of Gothenburg, Sweden

*Correspondence: llya Korsunskiy

iliakors@gmail.com

Daniel Munblit

daniel.munblit08@imperial.ac.uk

Specialty section:

This article was submitted to

Primary Immunodeficiencies,

a section of the journal

Frontiers in Immunology

Received: 08 October 2019 Accepted: 10 February 2020

Published: 03 March 2020

Citation:

Korsunskiy I, Blyuss O, Gordukova M, Davydova N, Zaikin A, Zinovieva N. Zimin S, Molchanov R, Salpagarova A, Eremeeva A, Filipenko M, Prodeus A, Korsunskiy A, Hsu P and Munblit D (2020) Expanding TREC and KREC Utility in Primary Immunodeficiency Diseases Diagnosis

Front. Immunol. 11:320

doi: 10.3389/fimmu.2020.00320

\section{Expanding TREC and KREC Utility in Primary Immunodeficiency Diseases Diagnosis}

\author{
Ilya Korsunskiy ${ }^{1,2 *}$, Oleg Blyuss ${ }^{2,3,4}$, Maria Gordukova ${ }^{1}$, Natalia Davydova ${ }^{1}$, Alexey Zaikin ${ }^{5}$, \\ Natalia Zinovieva ${ }^{1}$, Sergey Zimin ${ }^{1}$, Robert Molchanov ${ }^{6}$, Aminat Salpagarova ${ }^{2}$, \\ Alina Eremeeva ${ }^{2}$, Maxim Filipenko ${ }^{7}$, Andrey Prodeus ${ }^{1}$, Anatoliy Korsunskiy ${ }^{1,2}$, \\ Peter Hsu ${ }^{8,9,10}$ and Daniel Munblit ${ }^{2,9,11,12 \star}$ \\ ${ }^{1}$ Speransky Children's Hospital, Moscow, Russia, ${ }^{2}$ Department of Paediatrics and Paediatric Infectious Diseases, Institute of \\ Child's Health, Sechenov First Moscow State Medical University (Sechenov University), Moscow, Russia, ${ }^{3}$ Wolfson Institute \\ of Preventive Medicine, Queen Mary University of London, London, United Kingdom, ${ }^{4}$ School of Physics, Astronomy and \\ Mathematics, University of Hertfordshire, Hatfield, United Kingdom, ${ }^{5}$ Department of Mathematics and Institute for Women's \\ Health, University College London, London, United Kingdom, ${ }^{6}$ State Institution "Dnipropetrovsk Medical Academy of the \\ Ministry of Health of Ukraine", Dnipro, Ukraine, 'Pharmacogenomic Laboratory, Institute of Chemical Biology and \\ Fundamental Medicine, Novosibirsk, Russia, ${ }^{8}$ Allergy and Immunology, The Kids Research Institute, The Children's Hospital \\ at Westmead, Sydney, NSW, Australia, ${ }^{9}$ The In-vivo Global Network, an Affiliate of the World Universities Network (WUN), \\ New York, NY, United States, ${ }^{10}$ Discipline of Child and Adolescent Health, The University of Sydney, Sydney, NSW, Australia, \\ ${ }^{11}$ Inflammation, Repair and Development Section, Faculty of Medicine, NHLI, Imperial College London, London, \\ United Kingdom, ${ }^{12}$ Solov'ev Research and Clinical Center for Neuropsychiatry, Moscow, Russia
}

Primary immunodeficiency diseases (PID) area heterogeneous group of disorders caused by genetic defects of the immune system, which manifest clinically as recurrent infections, autoimmune diseases or malignancies. Early detection of PID remains a challenge, particularly in older children with milder and less specific symptoms. This study aimed to assess TREC and KREC diagnostic ability in PID. Data from children assessed by clinical immunologists at Speransky Children's Hospital, Moscow, Russia with suspected immunodeficiencies were analyzed between May 2013 and August 2016. Peripheral blood samples were sent for TREC/KREC, flow cytometry (CD3, CD4, CD8 and $\mathrm{CD19}$ ), IgA and IgG analysis. A total of 434 children [189 healthy, 97 with group I and II PID (combined T and B cell immunodeficiencies \& well-defined syndromes with immunodeficiency) and 148 group III PID (predominantly antibody deficiencies)] were included. Area under the curve (AUC) for TREC in PID groups I and II diagnosis reached $0.82(\mathrm{Cl}=0.75-0.90)$, with best model providing sensitivity of $65 \%$ and specificity of 92\%. Neither TREC, nor KREC had added value in PID group III diagnosis. In this study, the predictive value of TREC and KREC in PID diagnosis was examined. We found that the TREC had some diagnostic utility for groups I and II PID. Possibly, addition of TREC measurements to existing clinical diagnostic algorithms may improve their predictive value. Further investigations on a larger cohort are needed to evaluate TREC/KREC abilities to be used as diagnostic tools on a wider scale.

Keywords: TREC, KREC, primary immunodeficiency diseases, PID, primary immunodeficiency diseases diagnosis 


\section{HIGHLIGHTS}

\section{- What is already known about this topic?}

Assessment of TREC levels is actively used in the screening of severe combined immunodeficiency disorders (SCID).

\section{- What does this article add to our knowledge?}

This study shows that TREC may have a place not just in SCID screening but in the diagnosis of PID.

- How does this study impact current management guidelines? Evidence suggests that TREC may be a good addition to already existing diagnostic methods in groups I and II PID diagnosis. It may be particularly useful in less affluent environments with lack of access to flow cytometry.

\section{INTRODUCTION}

Primary immunodeficiency diseases (PID) are a heterogeneous group of disorders caused by genetic defects of the immune system, which manifest clinically as recurrent infections, autoimmune diseases or malignancies. The most severe forms of PID, severe combined immune deficiency (SCID) is intensively studied and has been found to be associated with fatal consequences in the first 2 years of life $(1,2)$.

Most forms of SCID can be detected by measuring the levels of T-cell recombination excision circles (TREC) in dried blood spots using real-time polymerase chain reaction (PCR) (3), while kappa-deleting recombination excision circles (KREC) are used to screen for agammaglobulinemia (4). TREC is a by-product of the T-cell receptor gene recombination, and KREC is a byproduct of the B-cell receptor recombination. Low levels of these molecules in $\mathrm{T}$ - and B-cells in peripheral blood were shown to be associated with T- and/or B-cell lymphopenia (4). The best possible outcome for patients with SCID can be achieved by timely hematopoietic stem cell transplantation or gene therapy before the development of infectious complications $(5,6)$, while early diagnosis is associated with a significant increase in treatment effectiveness (5). In patients with agammaglobunemia, the best outcome is achieved by initiating replacement therapy using intravenous or subcutaneous immunoglobulins.

PID influence child health-related quality of life, limiting physical, emotional, social and school functioning (7). Therefore, early detection of not only SCID but all PID patients is vital to improve the chances of appropriate management, in order to significantly reduce potential complications and improve life quality $(6,8)$.

At present, early detection of PID remains a challenge. This is particularly true in older children and in adults, potentially due to milder and less specific symptoms, a low level of awareness of PID amongst clinicians, as well as unavailability of necessary

Abbreviations: AUC, Area under receiver operating characteristic curve; CD, cluster of differentiation; CVID, Common Variable Immunodeficiency; DNA, Deoxyribonucleic acid; ICD-10, International Classification of Diseases, 10th Revision; Ig, immunoglobulin; IUIS, International Union of Immunological Societies; KREC, kappa-deleting element recombination circle; PCR, polymerase chain reaction; PID, Primary immunodeficiency diseases; ROC, Receiver Operating Curve; SCID, Severe Combined Immune Deficiency; TREC, T-cell recombination excision circles. diagnostic devices such as flow cytometry in hospital laboratories $(9,10)$. In some countries flow cytometry is not readily available and TREC/KREC analysis may represent a feasible alternative. It is therefore imperative to not only allow diagnosis of patients in low-resource facilities but also develop more cost-effective alternatives. Many studies showed that dried blood spots are robust (11) and useful as a potential alternative sample source for clinical purposes, epidemiological studies, and biobanking (12).

Flow cytometry is a more commonly used but more expensive diagnostic technique for PID detection, when compared with PCR (13). It requires a significant amount of training in highly specialized tertiary centers and therefore cannot be used as a screening tool.

Potential applications of TREC/KREC analysis were highlighted in the reviews by van Zelm and co-authors $(14,15)$. These included support therapy monitoring, patient classification and newborn screening for PID. Apart from apparent clinical benefits, assessment of B- and T- cell neogenesis in PID patients following stem cell transplantation (16) and KREC assessment in patients presenting with abnormalities in B-cell subsets to explain B-cell compartment aberrancies (17) may improve current state of knowledge.

This pilot study aims to assess diagnostic accuracy of TREC and KREC in children from 0 to 18 years of age with suspected PID.

\section{METHODS}

\section{Study Setting, Eligibility Criteria, and Ethics}

In this prospective study, we recruited all children referred by primary care physicians (polyclinic pediatricians) to a tertiary level center (Moscow City Pediatric Hospital \#9 named after Speransky, Moscow, Russia) with suspected immunodeficiencies and assessed by board-certified clinical immunologists between May 2013 and August 2016. The investigations and sample collection were conducted following ethical approval by the Speransky Children's Hospital Ethics Committee. Parental written consent was obtained for all participants as part of routine procedure at Speransky Children's Hospital. Parents/guardians were informed of the procedures in lay terms. The study design has been described in detail elsewhere (18).

\section{Outcome Definition}

The primary outcome of interest in this study was PID. We considered that a child has a PID if he or she had PID diagnosed by a physician. The diagnosis of different groups of PID was based on IUIS Phenotypic Classification for Primary Immunodeficiencies (19): group I was defined as immunodeficiencies affecting cellular and humoral immunity; group II corresponded to combined immunodeficiencies with associated or syndromic features, group III was defined as predominantly antibody deficiencies.

\section{Sample Analysis}

Peripheral blood samples were taken by venipuncture during morning hours, aliquoted and sent for complete blood count, flow cytometry, immunoglobulin (IgA, IgG), and TREC/KREC 
analysis. All blood samples were EDTA-anticoagulated and analyzed on the day of collection to avoid cellular death. Immunoglobulin levels were measured in blood serum.

Sample analysis was performed as described elsewhere (18). In brief, three-four color flow cytometric immunophenotyping with directly labeled monoclonal antibodies was used to determine the following immune cell subsets: CD3-CD19+, CD3-CD $(16+$ $56)+, \mathrm{CD} 3+\mathrm{CD} 4+$, and $\mathrm{CD} 3+\mathrm{CD} 8+$ following manufacturer's protocol. Further analysis was performed with a FACS Canto II flow cytometer using FACSDiva v7.0 software (Becton Dickinson). The total leucocyte count and differential was measured with Advia 2120i hematology analyzer (Siemens). The absolute size of each lymphocyte subpopulation was calculated by multiplying the relative size of the lymphocyte subpopulation by the absolute lymphocyte count. Immunoglobulin levels were assessed using a biochemical analyzer Architect C8000 (Abbott, USA, Abbott kits) in accordance with manufacturers' protocol. TREC and KREC assays were performed using realtime PCR with fluorescent hybridization probes and reagents for TREC/KREC assays: T\&B PCR kit (ABV-test, Russia) (20), in whole blood. The TREC/KREC levels were assayed in whole blood samples as described previously $(16,18,20)$, In brief, DNA was extracted from $100 \mu \mathrm{l}$ EDTA anticoagulated whole blood by using RIBO-prep nucleic acid extraction kit (Amplisense ${ }^{\circledR}$,
Russia). The Real-time qPCR was performed using CFX 96 Real-Time PCR System (Bio Rad, USA). Amplification of ALB was used to assess correct sampling and quality of DNA extraction, as well as to determine TREC and KREC levels. The number of TREC/KREC copies was calculated per $10^{5}$ white blood cells, accounting for the quantity of ALB using the formula: [The number of TREC/KREC copies/the number of ALB copies] $\times 200,000$. The normal/cutoff levels of TRECs and KRECs of 1,000 copies $/ 10^{5}$ cells were used.

\section{Statistical Analysis}

Shapiro-Wilk test was used to assess whether analyzed variables were normally distributed. Since the null hypothesis was not rejected, Spearmen correlation coefficient was used to assess the strength of the correlation between the variables. Sensitivity, specificity and their 95\% confidence intervals were computed with stratified bootstrap replicates (21). Area under Receiver Operating Characteristic (ROC) - curve (AUC) calculation was followed by $95 \%$ confidence interval as suggested by DeLong et al. (22). The diagnostic accuracy measures used were: sensitivity, specificity, positive predictive value (PPV) and negative predictive value (NPV). TREC/KREC accuracy in diagnosing group I/II and group III PIDs were assessed. The ROC-analysis was performed separately for PID groups I/II and

TABLE 1 | Characteristics of study participants.

\begin{tabular}{|c|c|c|c|c|c|}
\hline \multirow[t]{2}{*}{ PID Group } & \multirow[t]{2}{*}{ Total number of patients } & \multirow{2}{*}{$\begin{array}{l}\text { Clinical diagnosis } \\
\text { (number of patients) }\end{array}$} & \multirow[t]{2}{*}{ Age } & \multicolumn{2}{|c|}{ Gender } \\
\hline & & & & Male & Female \\
\hline $\begin{array}{l}\text { Group I } \\
\text { ("Combined PID") } \\
\text { Immunodeficiencies } \\
\text { affecting cellular and } \\
\text { humoral immunity }\end{array}$ & 17 & D81 Combined immunodeficiencies (17) & $\begin{array}{l}0-12 \text { months } \\
1-6 \text { years } \\
6-12 \text { years } \\
12-18 \text { years }\end{array}$ & $\begin{array}{l}9 \\
2 \\
1 \\
0\end{array}$ & $\begin{array}{l}5 \\
0 \\
0 \\
0\end{array}$ \\
\hline $\begin{array}{l}\text { Group II } \\
\text { ("Syndromic PID") } \\
\text { CID with associated or } \\
\text { syndromic features }\end{array}$ & 80 & $\begin{array}{l}\text { D82 Immunodeficiency associated with other } \\
\text { major defects (13) } \\
\text { D82.1 Di George syndrome (39) } \\
\text { D82.4 Hyperimmunoglobulin E syndrome (5) } \\
\text { D84.8 Other specified immunodeficiencies (15) } \\
\text { G11.3 (8) }\end{array}$ & $\begin{array}{l}0-12 \text { months } \\
1-6 \text { years } \\
6-12 \text { years } \\
12-18 \text { years }\end{array}$ & $\begin{array}{c}7 \\
26 \\
10 \\
4\end{array}$ & $\begin{array}{c}9 \\
17 \\
6 \\
3\end{array}$ \\
\hline $\begin{array}{l}\text { Group III } \\
\text { ("Antibody PID") } \\
\text { Predominantly antibody } \\
\text { deficiencies }\end{array}$ & 148 & $\begin{array}{l}\text { D80.0 Immunodeficiency with predominantly } \\
\text { antibody defects (4) } \\
\text { D80.1 Non-familial hypogammaglobulinaemia } \\
\text { (47) } \\
\text { D80.2 Selective deficiency of immunoglobulin A } \\
\text { (34) } \\
\text { D80.3 Selective deficiency of immunoglobulin G } \\
\text { (24) } \\
\text { D80.4 Selective deficiency of immunoglobulin M } \\
\text { (1) } \\
\text { D80.5 Immunodeficiency with increased } \\
\text { immunoglobulin M (4) } \\
\text { D83 Common variable immunodeficiency (34) }\end{array}$ & $\begin{array}{l}0-12 \text { months } \\
1-6 \text { years } \\
6-12 \text { years } \\
12-18 \text { years }\end{array}$ & $\begin{array}{c}6 \\
21 \\
28 \\
38\end{array}$ & $\begin{array}{c}2 \\
17 \\
21 \\
13\end{array}$ \\
\hline $\begin{array}{l}\text { Control group (Healthy } \\
\text { children) }\end{array}$ & 226 & No clinical diagnosis of PID & $\begin{array}{l}0-12 \text { months } \\
1-6 \text { years } \\
6-12 \text { years } \\
12-18 \text { years }\end{array}$ & $\begin{array}{c}9 \\
41 \\
36 \\
25\end{array}$ & $\begin{array}{c}5 \\
48 \\
33 \\
29\end{array}$ \\
\hline
\end{tabular}

All codes and diagnoses are in accordance with international classification of diseases, 10th revision (ICD-10). 
PID group III. The performance characteristics of all lymphocyte subpopulations as well as combinations of TREC and KREC were evaluated and compared in terms of (a) the sensitivity (proportion detected of those with PID) at a fixed specificity (proportion of controls correctly detected not to have PID) and (b) AUC.

Due to TREC levels decreasing with age, every TREC measurement was divided by the corresponding TREC reference interval for the patient's age group prior to analysis.

Results were considered statistically significant if $p$-value was smaller than 0.05 . All calculations were done using $\mathrm{R}$ package version 3.4.1.

\section{RESULTS}

\section{Study Population}

The data was extracted from clinical notes and the laboratory database of Speransky Children's Hospital. Out of 3,055 patients requiring flow cytometry within the given period of time, due to financial restrictions (those eligible for flow cytometry to be covered by compulsory health insurance in accordance to local regulations. Regulations did not change throughout the study period.), a total of 839 samples were analyzed using flow cytometry and TREC assay and 931 samples were analyzed using flow cytometry and KREC assay. Data on confirmed clinical diagnosis was available from 471 participants. All data points required for TREC/KREC diagnostic properties assessment were available from 434 children and were included in the statistical analysis. Out of 434 children with a doctor's confirmed diagnosis, 189 were immunologically healthy, 97 were group I and II PID patients and 148 were group III PID patients. The following conditions were diagnosed in each subcategory in accordance to International Classification of Diseases, 10th revision (ICD10) and classified following IUIS Phenotypic Classification for Primary Immunodeficiencies (19): group I (combined immunodeficiencies), group II (immunodeficiency associated with other major defects, ataxia telangiectasia and other specified immunodeficiencies), group III (immunodeficiency
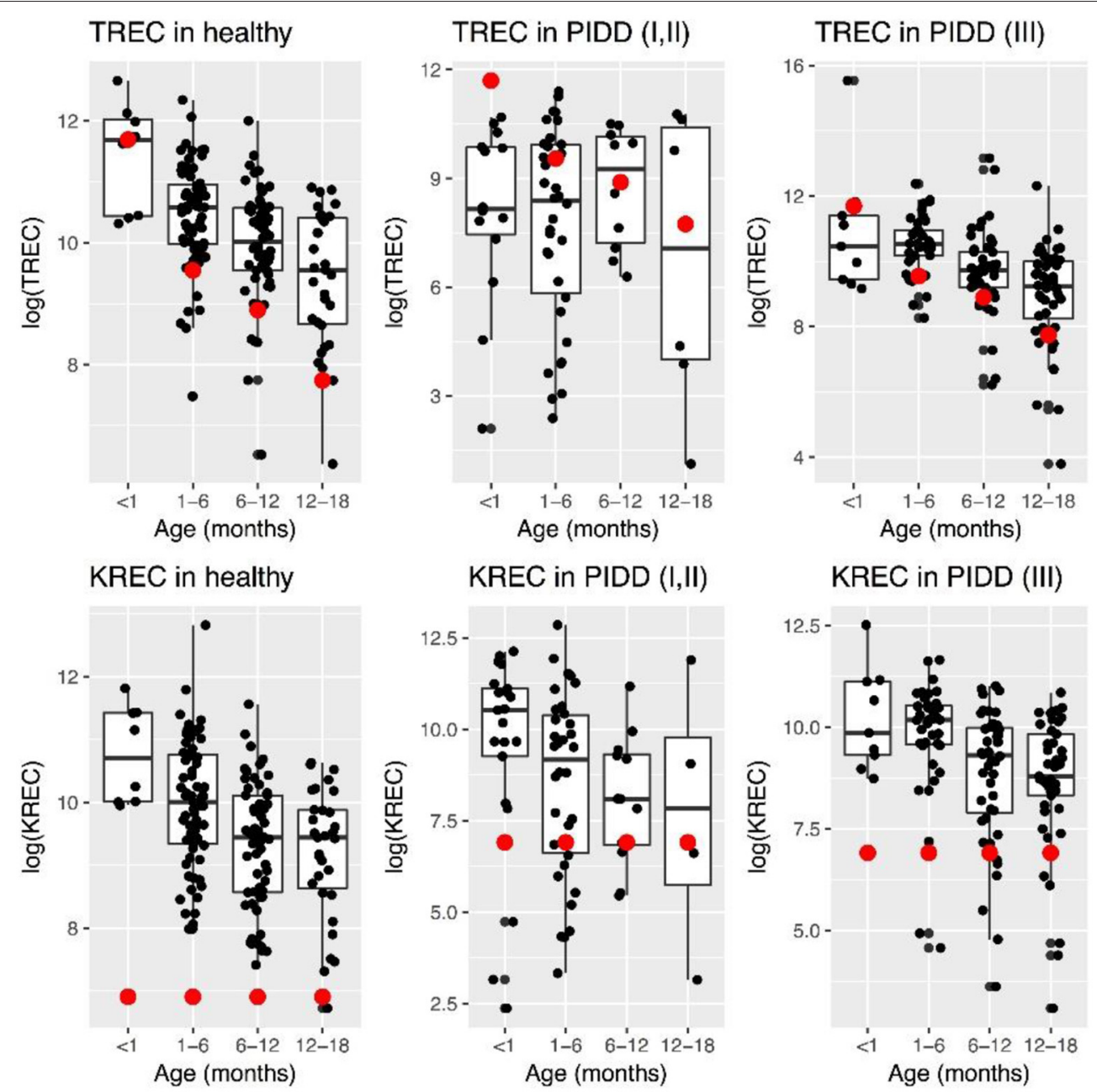

FIGURE 1 | Patterns of change in TREC and KREC log-transformed (natural logarithm) levels in healthy individuals and PID patients at different age. Red dot represents minimal normal level for a given age group. 


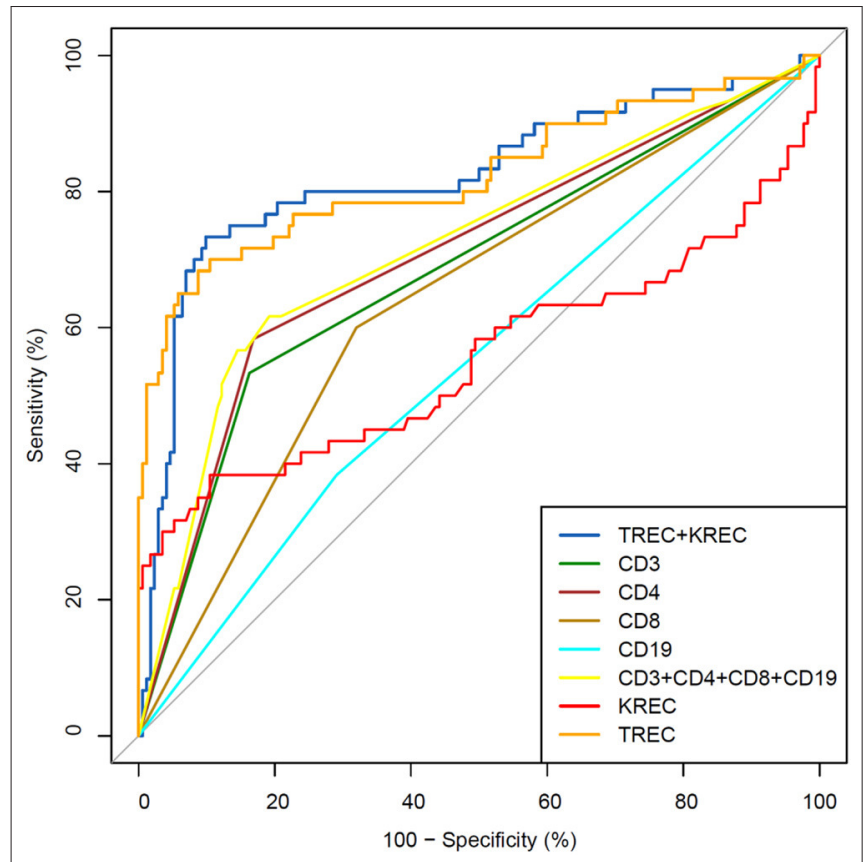

FIGURE 2 | Receiver operating characteristic (ROC) curves for each of lymphocyte subpopulations (CD3, CD4, CD8, and CD19) individually; combined diagnostic ability of all lymphocyte subpopulations and diagnostic ability of TREC and KREC combination in "Combined PID" and "Syndromic PID" diagnosis. Healthy individuals ( $n=172$ ); children diagnosed with "Combined PID" and "Syndromic PID" $(n=60)$. AUC for TREC and a combination of TREC and $\mathrm{KREC}=0.82(95 \% \mathrm{Cl}=0.75-0.90)$.

with predominantly antibody defects and common variable immunodeficiency). For the sake of the readers' convenience we will use the following terminology in the following sections of the manuscript: "Combined PID" for group I, "Syndromic PID” for group II and "Antibody PID” for group III. The diagnoses were reached using clinical signs and immune phenotype. Genetic testing was not available at the recruitment site.

All the samples were analyzed using flow cytometry, turbidimetry, and TREC/KREC assays and were included into the primary analysis of this study. Demographic data of the participants is presented in Table $\mathbf{1}$.

\section{Descriptive Results of Flow Cytometry and TREC/KREC Testing}

Levels of TREC decreased with age in healthy children, while this was less evident in "Combined PID" and "Syndromic PID." Overall TREC levels were lower in "Combined PID” and "Syndromic PID" compared to healthy individuals (Figure 1).

\section{TREC/KREC Diagnostic Accuracy}

The area under the curve for lymphocyte subpopulations (CD 3, 4, 8, and 19), immunoglobulins (IgA, IgG) and TREC/KREC levels were assessed. Separate analyses were undertaken for "Combined PID" and "Syndromic PID" (Figure 2) and “Antibody PID” (Figure 3). The same analysis was performed to assess ability of TREC/KREC to differentiate between "Combined PID," "Syndromic PID," and “Antibody PID."

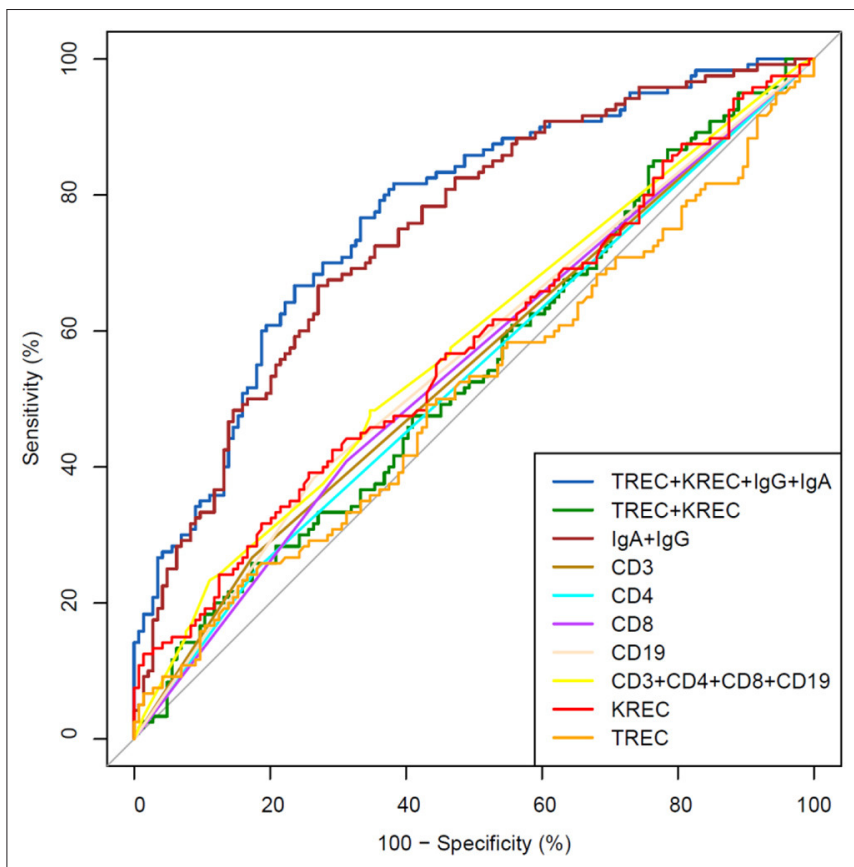

FIGURE 3 | Receiver operating characteristic (ROC) curves for each of lymphocyte subpopulations (CD3, CD4, CD8, and CD19) individually; combined diagnostic ability of all lymphocyte subpopulations; IgA and IgG combined; TREC and KREC combined and diagnostic ability of TREC, KREC, IgA, and IgG combination in "Antibody PID" diagnosis. Healthy individuals $(n=$ 144); children diagnosed with "Antibody PID" $(n=120)$. IgA, IgG, TREC, and KREC AUC $=0.77(95 \% \mathrm{Cl}=0.71-0.82)$.

TABLE 2 | Diagnostic accuracy measures for different cutoff points of the predicted probabilities for TREC in "Combined PID" and "Syndromic PID" diagnosis.

\begin{tabular}{lccccc}
\hline $\begin{array}{l}\text { Cutoff point } \\
\text { (probability) }\end{array}$ & PPV (\%) & NPV (\%) & Sensitivity (\%) & $\begin{array}{c}\text { Specificity } \\
\text { (\%) }\end{array}$ & $\begin{array}{c}\text { Youden } \\
\text { index }\end{array}$ \\
\hline 0.05 & 28 & 92 & 97 & 13 & 9.5 \\
0.1 & 29 & 90 & 93 & 21 & 14.2 \\
0.15 & 32 & 90 & 90 & 32 & 22 \\
0.2 & 33 & 89 & 85 & 41 & 25.7 \\
0.25 & 37 & 88 & 78 & 54 & 31.8 \\
0.3 & 46 & 90 & 78 & 67 & 45.7 \\
0.35 & 59 & 89 & 72 & 83 & 54.3 \\
$\mathbf{0 . 4}$ & $\mathbf{7 5}$ & $\mathbf{8 8}$ & $\mathbf{6 5}$ & $\mathbf{9 2}$ & $\mathbf{5 7 . 4}$ \\
\hline
\end{tabular}

Optimum cut-off point based on maximum value of the $\mathrm{J}$ index is presented in bold.

AUC for TREC and a combination of TREC and KREC for "Combined PID" and "Syndromic PID" diagnosis was 0.82 (95\% $\mathrm{CI}=0.75-0.90)$. As KREC did not add value to the model's predictive capacity, the data for TREC is presented (Table 2). The cutoff point of a probability of 0.4 showed the best diagnostic accuracy with regards to the sensitivity and specificity (65 and $92 \%), J=57.4$.

A combination of IgA, IgG, TREC and KREC demonstrated the best AUC 0.77 (95\% CI = 0.71-0.82) for "Antibody PID" diagnosis but neither TREC or KREC nor combination of two 
TABLE 3 | Diagnostic accuracy measures for different cutoff points of the predicted probabilities for a combination of TREC and KREC in "Antibody PID" diagnosis

\begin{tabular}{lccccc}
\hline $\begin{array}{l}\text { Cutoff point } \\
\text { (probability) }\end{array}$ & PPV (\%) & NPV (\%) & Sensitivity (\%) & $\begin{array}{c}\text { Specificity } \\
\text { (\%) }\end{array}$ & $\begin{array}{c}\text { Youden } \\
\text { index }\end{array}$ \\
\hline 0.35 & 46 & 75 & 98 & 4 & 2.5 \\
0.4 & 46 & 64 & 93 & 11 & 3.6 \\
0.45 & 47 & 56 & 61 & 42 & 2.5 \\
$\mathbf{0 . 5}$ & $\mathbf{5 7}$ & $\mathbf{5 6}$ & $\mathbf{1 4}$ & $\mathbf{9 1}$ & $\mathbf{5 . 2}$ \\
0.55 & 42 & 54 & 4 & 95 & 0 \\
0.6 & 50 & 55 & 2 & 99 & 0.3 \\
\hline
\end{tabular}

Optimum cut-off point based on maximum value of the $\mathrm{J}$ index is presented in bold.

yielded good diagnostic ability (AUC 0.54, 95\% CI = 0.47-0.61), Table 3. TREC and KREC combination did not demonstrate any added value to immunoglobulin level detection.

We then performed analysis to study ability of TREC/KREC to differentiate between "Combined PID," "Syndromic PID," and "Antibody PID." As KREC did not add value to the predictive model, the predictive model based on TREC levels is presented (Figure 4). The AUC for TREC in "Combined PID" and "Syndromic PID" diagnosis was 0.79 (95\% CI = 0.70-0.87). A cutoff point of a probability of 0.4 showed the best diagnostic accuracy with regards to the sensitivity and specificity (66 and $84 \%$; (Table 4), $J=50.2$.

\section{DISCUSSION}

In this study, we tested the accuracy of TREC and KREC in PID diagnosis. The models showed decent utility of TREC in the diagnosis of "Combined PID" and "Syndromic PID." To our knowledge, this is the first attempt to use TREC/KREC not in SCID screening but in PID diagnostics.

PID is a large group of disorders encompassing more than 400 conditions affecting development and/or functioning of the immune system (23). Flow cytometry is a sensitive and important tool in evaluating the immune system function and in PID diagnosis (24). However, it is expensive, not easily available in developing countries and requires appropriate training and equipment. TREC and KREC may represent cheaper alternatives and/or add value to PID diagnosis and screening. Low cost methodology can be used in small laboratories and rural settings, where complex and expensive tools are unavailable, to provide access to primary PID evaluation.

Prior studies have noted the need in screening tool for early SCID diagnosis, to reduce the risk of infections and organ damage $(6,25)$. Early diagnosis is particularly important as lack of early treatment is associated with severe complications and increased mortality rates (26). TREC is a common screening approach used for early SCID detection around the globe, providing a good combination of sensitivity and specificity $(27,28)$ with high cost-effectiveness (29). KREC's role in early screening is still debatable but some data suggest that it may add value in certain cases (30). While TREC's indispensability in SCID screening is obvious, very little can be found in the literature on the question of TREC/KREC use in other PID

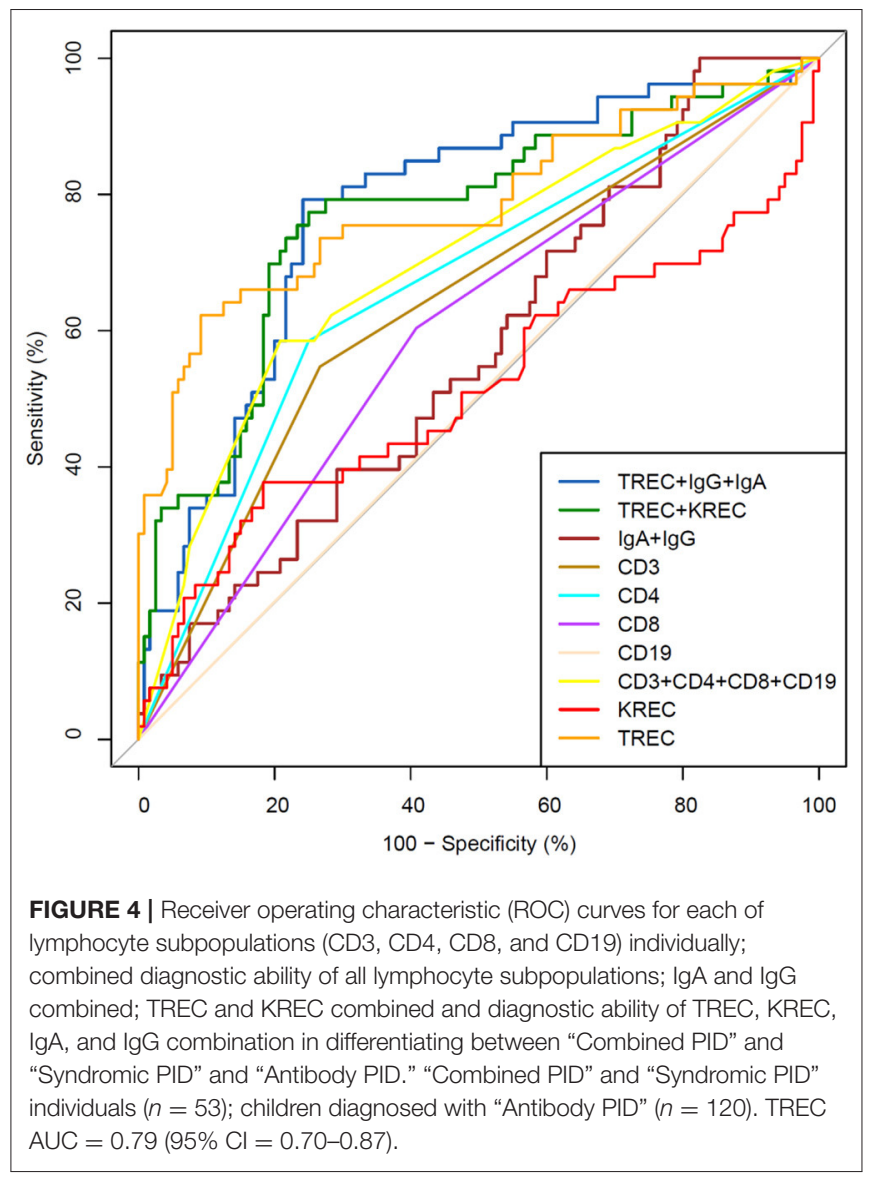

TABLE 4 | Differential diagnosis for "combined PID" and "syndromic PID" and "antibody PID." Accuracy measures for different cutoff points of the predicted probabilities for TREC.

\begin{tabular}{lccccc}
\hline $\begin{array}{l}\text { Cutoff point } \\
\text { (probability) }\end{array}$ & PPV (\%) & NPV (\%) & Sensitivity (\%) & $\begin{array}{c}\text { Specificity } \\
\text { (\%) }\end{array}$ & $\begin{array}{c}\text { Youden } \\
\text { index }\end{array}$ \\
\hline 0.05 & 31 & 80 & 96 & 7 & 2.9 \\
0.1 & 33 & 89 & 96 & 13 & 9.5 \\
0.15 & 34 & 88 & 94 & 18 & 12.6 \\
0.2 & 36 & 89 & 93 & 28 & 20 \\
0.25 & 38 & 88 & 89 & 35 & 23.7 \\
0.3 & 40 & 84 & 79 & 47 & 25.9 \\
0.35 & 51 & 86 & 76 & 68 & 43.8 \\
$\mathbf{0 . 4}$ & $\mathbf{6 5}$ & $\mathbf{8 5}$ & $\mathbf{6 6}$ & $\mathbf{8 4}$ & $\mathbf{5 0 . 2}$ \\
\hline
\end{tabular}

Optimum cut-off point based on maximum value of the $J$ index is presented in bold.

diagnosis or their use as a screening tool (31-33). Previous attempts to this end have predominantly focused on Common Variable Immunodeficiency (CVID).

We hypothesized, that TREC and KREC may be adopted as a surrogate method of PID diagnosis. Our models demonstrated good AUCs indicating the potential of TREC to be used as an additional tool in PID diagnosis. Our data show that when TREC is used for differentiation between "Combined PID" and "Syndromic PID" patients and healthy individuals, the cut-off point probability of 0.4 provides high specificity (92\%) with 
acceptable sensitivity (65\%), further supporting our hypothesis that use of TREC may have its place in PID diagnosis. TREC may serve as an addition to existing tests and may be used as a prerequisite to flow cytometry. We did not use any clinical questionnaires in this study but expect that laboratory findings combined with additional clinical data using standardized instruments may facilitate development of a stronger diagnostic model, improving utility.

TREC and KREC whether individually or combined, did not show good diagnostic ability in diagnosing "Antibody PID.” However, this result was expected, with immunoglobulin measurement having the primary role in aiding the diagnosis. Our further analysis highlights potential for TREC level assessment in discrimination between "Combined PID," "Syndromic PID," and "Antibody PID." When a cut-off of 0.4-point probability was used, TREC reached specificity of $84 \%$. At present, the wide variety and lack of specificity of PID clinical manifestations, do not allow physicians to determine the exact area of immune defect during initial clinical examination. Thus, the extensiveness of the primary laboratory examination in a patient with suspected PID is often determined by subjective clinical criteria, based on physician expertise. Laboratory tests normally used vary from immunoglobulin level assessment to assessment of a wide range of lymphocyte subpopulations. There are no predefined universal guidelines on how detailed the laboratory analysis should be, while selection of diagnostic tests depends on the given clinical settings and clinical immunologist. Normally, if initial tests reveal deviations in humoral immunity the next logical step is cellular immunity assessment to exclude combined immunodeficiency and diagnose "Antibody PID." Our data suggests that TREC appears to be a useful additional tool to aid in the differentiation between combined immunodeficiency and antibody deficiencies, particularly in the settings with limited access to flow cytometry. The qualitative method of TREC/KREC assessment is easy and can be applied equally well to both whole blood and Guthrie cards, as DNA extraction can be performed on either of these samples.

A few diagnostic algorithms/approaches to children with suspected PID were proposed. Among them excellent algorithm from Dutch immunologist Esther DeVries', based on a pattern recognition approach and decision trees (34), and the Jeffrey Modell Foundation's 4 steps (35). We do not propose changes to existing clinical approaches in routine PID diagnosis and fully acknowledge that TREC/KREC should not be used as a replacement of flow cytometry. Addition of TREC/KREC measurements to aforementioned clinical approaches, nevertheless, may improve predictive capacity of the tools, which is worth further investigation.

The main limitation of this study is related to the use of ICD10 classification for PID diagnosis. The same ICD-10 code may sometimes include heterogenous group of immunodeficiencies. Genetic testing would be a preferable option; however, this was not available for most of the patients due to economical restrictions. Another limitation is the lack of Guthrie card use in our study with all samples analyzed using whole blood, which does not allow for a result extrapolation. No difference is expected, however, between DNA extraction from the whole blood sample and dried blood spot. Recruitment of patients of three PID groups only, may also be considered a limitation, even though it is unlikely to have influenced the outcomes of this study. We acknowledge that participants defined as "immunologically healthy" in this study can be considered as "healthy" to a high degree of certainty, but no information on naive cells and memory cells were collected. It is clear, however, that "immunologically healthy" participants in this study do not belong to Group I ("Combined PID"). Our study would also have benefited from assessment of TREC/KREC diagnostic accuracy in patients with a particular subtype of PID [e.g., Xlinked agammaglobulinemia (XLA)], but it was not possible due to restricted number of patients. This should be addressed in future research.

In conclusion, we found evidence that TREC may have a place in aiding PID diagnosis. The models showed decent diagnostic accuracy measures for TREC in diagnosing "Combined PID" and "Syndromic PID". Further investigations in a larger cohort in combination with addition of genetic diagnoses and/or questionnaires focused on clinical symptoms are needed to improve diagnostic performance and to further evaluate TREC potential on a wider scale. It is too premature to draw definitive conclusions, but with a few diagnostic algorithms available (e.g., Esther DeVries' and Jeffrey Modell Foundation's 4 steps), addition of TREC to such algorithms may allow for an improved predictive ability. We would like to stress that TREC-based PID diagnosis may be particularly important in the recourselimited countries and further research will benefit children in these settings.

\section{DATA AVAILABILITY STATEMENT}

The datasets generated for this study are available on request to the corresponding author.

\section{ETHICS STATEMENT}

The studies involving human participants were reviewed and approved by Speransky Children's Hospital Ethics Committee. Written informed consent to participate in this study was provided by the participants' legal guardian/next of kin.

\section{AUTHOR CONTRIBUTIONS}

IK, MF, AP, AK, and DM conceived and designed the experiments and study analysis. MG and ND performed the experiments. IK, $\mathrm{NZ}$, SZ, and AS collected, extracted, and sorted the data. OB, $\mathrm{RM}$, and $\mathrm{AZ}$ analyzed the data. AE reviewed additional available evidence on the matter. IK, $\mathrm{PH}$, and DM wrote the manuscript.

\section{FUNDING}

AZ acknowledges support by the MRC grant MR/R02524X/1.

\section{ACKNOWLEDGMENTS}

Authors are very grateful to Loukia Petrou for English Language editing. 


\section{REFERENCES}

1. Dvorak CC, Cowan MJ, Logan BR, Notarangelo LD, Griffith LM, Puck JM, et al. The natural history of children with severe combined immunodeficiency: baseline features of the first fifty patients of the primary immune deficiency treatment consortium prospective study 6901. J Clin Immunol. (2013) 33:1156-64. doi: 10.1007/s10875-013-9917-y

2. Yao CM, Han XH, Zhang YD, Zhang H, Jin YY, Cao RM, et al. Clinical characteristics and genetic profiles of 44 patients with severe combined immunodeficiency (SCID): report from Shanghai, China (20042011). J Clin Immunol. (2013) 33:526-39. doi: 10.1007/s10875-0129854-1

3. Chan K, Puck JM. Development of population-based newborn screening for severe combined immunodeficiency. J Allergy Clin Immunol. (2005) 115:391-8. doi: 10.1016/j.jaci.2004. 10.012

4. Serana F, Chiarini M, Zanotti C, Sottini A, Bertoli D, Bosio A, et al. Use of $\mathrm{V}(\mathrm{D}) \mathrm{J}$ recombination excision circles to identify T- and B-cell defects and to monitor the treatment in primary and acquired immunodeficiencies. J Transl Med. (2013) 11:119. doi: 10.1186/1479-5876-11-119

5. Pai SY, Logan BR, Griffith LM, Buckley RH, Parrott RE, Dvorak CC, et al. Transplantation outcomes for severe combined immunodeficiency, 20002009. N Engl J Med. (2014) 371:434-46. doi: 10.1056/NEJMoa1401177

6. Brown L, Xu-Bayford J, Allwood Z, Slatter M, Cant A, Davies EG, et al. Neonatal diagnosis of severe combined immunodeficiency leads to significantly improved survival outcome: the case for newborn screening. Blood. (2011) 117:3243-6. doi: 10.1182/blood-2010-08-3 00384

7. Peshko D, Kulbachinskaya E, Korsunskiy I, Kondrikova E, Pulvirenti F, Quinti I, et al. Health-related quality of life in children and adults with primary immunodeficiencies: a systematic review and meta-analysis. $J$ Allergy Clin Immunol Pract. (2019) 7:1929-57 e5. doi: 10.1016/j.jaip.2019. 02.013

8. Chan A, Scalchunes C, Boyle M, Puck JM. Early vs. delayed diagnosis of severe combined immunodeficiency: a family perspective survey. Clin Immunol. (2011) 138:3-8. doi: 10.1016/j.clim.2010. 09.010

9. Garcia JM, Gamboa P, de la Calle A, Hernandez MD, Caballero MT, Garcia BE, et al. Committee of immunology of the Spanish socierty of, and clinical I. diagnosis and management of immunodeficiencies in adults by allergologists. J Investig Allergol Clin Immunol. (2010) 20:185-94. Available online at: http:// www.jiaci.org/summary/vol20-issue3-num584.

10. Peter HH. Adult-onset immunodeficiency-why is it important in rheumatology? Arthritis Res Ther. (2013) 15:105. doi: 10.1186/ar4121

11. Rajatileka S, Luyt K, El-Bokle M, Williams M, Kemp H, Molnar E, et al. Isolation of human genomic DNA for genetic analysis from premature neonates: a comparison between newborn dried blood spots, whole blood and umbilical cord tissue. BMC Genet. (2013) 14:105. doi: 10.1186/1471-2156-14-105

12. Kumar A, Mhatre S, Godbole S, Jha P, Dikshit R. Optimization of extraction of genomic DNA from archived dried blood spot (DBS): potential application in epidemiological research \& bio banking. Gates Open Res. (2018) 2:57. doi: 10.12688/gatesopenres.12855.1

13. Puck JM, S.N.S.W. Group. Population-based newborn screening for severe combined immunodeficiency: steps toward implementation. J Allergy Clin Immunol. (2007) 120:760-8. doi: 10.1016/j.jaci.2007. 08.043

14. van Zelm MC, Szczepanski $\mathrm{T}$, van der Burg $\mathrm{M}$, van Dongen JJ. Replication history of B lymphocytes reveals homeostatic proliferation and extensive antigen-induced B cell expansion. J Exp Med. (2007) 204:645-55. doi: 10.1084/jem.200 60964

15. van Zelm MC, van der Burg M, Langerak AW, van Dongen JJ. PID comes full circle: applications of V(D)J recombination excision circles in research, diagnostics and newborn screening of primary immunodeficiency disorders. Front Immunol. (2011) 2:12. doi: 10.3389/fimmu.2011.00012

16. Sottini A, Ghidini C, Zanotti C, Chiarini M, Caimi L, Lanfranchi A, et al. Simultaneous quantification of recent thymic T-cell and bone marrow B-cell emigrants in patients with primary immunodeficiency undergone to stem cell transplantation. Clin Immunol. (2010) 136:217-27. doi: 10.1016/j.clim.2010.04.005

17. van der Burg $M$, Pac $M$, Berkowska MA, Goryluk-Kozakiewicz B, Wakulinska A, Dembowska-Baginska B, et al. Loss of juxtaposition of RAG-induced immunoglobulin DNA ends is implicated in the precursor B-cell differentiation defect in NBS patients. Blood. (2010) 115:4770-7. doi: 10.1182/blood-2009-10-250514

18. Korsunskiy I, Blyuss O, Gordukova M, Davydova N, Gordleeva $\mathrm{S}$, Molchanov R, et al. TREC and KREC levels as a predictors of lymphocyte subpopulations measured by flow cytometry. Front Physiol. (2018) 9:1877. doi: 10.3389/fphys.2018. 01877

19. Bousfiha A, Jeddane L, Picard C, Ailal F, Bobby Gaspar H, Al-Herz W, et al. The 2017 IUIS phenotypic classification for primary immunodeficiencies. J Clin Immunol. (2018) 38:129-43. doi: 10.1007/s10875-0170465-8

20. Deripapa E, Balashov D, Rodina Y, Laberko A, Myakova N, Davydova NV, et al. Prospective study of a cohort of Russian Nijmegen breakage syndrome patients demonstrating predictive value of low kappa-deleting recombination excision circle (KREC) numbers and beneficial effect of hematopoietic stem cell transplantation (HSCT). Front Immunol. (2017) 8:807. doi: 10.3389/fimmu.2017.00807

21. Carpenter J, Bithell J. Bootstrap confidence intervals: when, which, what? A practical guide for medical statisticians. Stat Med. (2000) 19:1141-64. doi: 10.1002/(SICI)1097-025819:9<;1141::AID-SIM479>;3.0.CO;2-F

22. DeLong ER, DeLong DM, Clarke-Pearson DL. Comparing the areas under two or more correlated receiver operating characteristic curves: a nonparametric approach. Biometrics. (1988) 44:837-45. doi: 10.2307/25 31595

23. Picard C, Al-Herz W, Bousfiha A, Casanova JL, Chatila T, Conley ME, et al. Primary immunodeficiency diseases: an update on the classification from the international union of immunological societies expert committee for primary immunodeficiency 2015. J Clin Immunol. (2015) 35:696-726. doi: 10.1007/s10875-015-0201-1

24. Kanegane H, Hoshino A, Okano T, Yasumi T, Wada T, Takada H, et al. Flow cytometry-based diagnosis of primary immunodeficiency diseases. Allergol Int. (2018) 67:43-54. doi: 10.1016/j.alit.2017. 06.003

25. Adeli MM, Buckley RH. Why newborn screening for severe combined immunodeficiency is essential: a case report. Pediatrics. (2010) 126:e465-9. doi: 10.1542/peds.2009-3659

26. Rozmus J, Junker A, Thibodeau ML, Grenier D, Turvey SE, Yacoub W, et al. Severe combined immunodeficiency (SCID) in Canadian children: a national surveillance study. J Clin Immunol. (2013) 33:1310-6. doi: 10.1007/s10875-0139952-8

27. Verbsky JW, Baker MW, Grossman WJ, Hintermeyer M, Dasu T, Bonacci $B$, et al. Newborn screening for severe combined immunodeficiency; the Wisconsin experience (2008-2011). J Clin Immunol. (2012) 32:82-8. doi: 10.1007/s10875-011-9609-4

28. van der Spek J, Groenwold RH, van der Burg M, van Montfrans JM. TREC based newborn screening for severe combined immunodeficiency disease: a systematic review. J Clin Immunol. (2015) 35:416-30. doi: 10.1007/s10875-015-0152-6

29. Ding Y, Thompson JD, Kobrynski L, Ojodu J, Zarbalian G, Grosse SD. Cost-effectiveness/cost-benefit analysis of newborn screening for severe combined immune deficiency in Washington State. J Pediatr. (2016) 172:127-35. doi: 10.1016/j.jpeds.2016. 01.029

30. Lodi L, Ricci S, Romano F, Ghiori F, Canessa C, Lippi F, et al. Newborn screening for PIDs using both TREC and KREC identifies late occurrence of B cells. Pediatr Allergy Immunol. (2017) 28:498-500. doi: 10.1111/ pai. 12733

31. Lee WI, Huang JL, Lin SJ, Yeh KW, Chen LC, Ou LS, et al. Applying T-cell receptor excision circles and immunoglobulin kappa-deleting recombination excision circles to patients with primary immunodeficiency diseases. Ann Med. (2014) 46:555-65. doi: 10.3109/07853890.2014.941920 
32. Kamae C, Nakagawa N, Sato H, Honma K, Mitsuiki N, Ohara O, et al. Common variable immunodeficiency classification by quantifying $\mathrm{T}$-cell receptor and immunoglobulin kappa-deleting recombination excision circles. J Allergy Clin Immunol. (2013) 131:1437-40 e5. doi: 10.1016/j.jaci.2012. 10.059

33. Atschekzei F, Ahmad F, Witte T, Jacobs R, Schmidt RE. Limitation of simultaneous analysis of $\mathrm{T}$-cell receptor and kappa-deleting recombination excision circles based on multiplex real-time polymerase chain reaction in common variable immunodeficiency patients. Int Arch Allergy Immunol. (2016) 171:136-40. doi: 10.1159/0004 50950

34. de Vries E, m. European Society for Immunodeficiencies. Patientcentred screening for primary immunodeficiency, a multi-stage diagnostic protocol designed for non-immunologists: 2011 update. Clin Exp Immunol. (2012) 167:108-19. doi: 10.1111/j.1365-2249.2011.0 4461.x

35. Jeffrey Modell Foundation. Four Stages of Testing for Primary Immunodeficiency. Jeffrey Modell Foundation (2016).
Conflict of Interest: MG, MF, and IK are board members for ABV-test. MG, MF, $\mathrm{AP}$, and IK has a patent with ABV-test. DM has given paid lectures for Merck Sharp \& Dohme (MSD) and Bayer. DM also is a member of ILSI Europe: Immune Competence Across Lifespan: Impact of Nutrition on Immune Competence and its Consequences Later in Life expert group.

The remaining authors declare that the research was conducted in the absence of any commercial or financial relationships that could be construed as a potential conflict of interest.

Copyright (C) 2020 Korsunskiy, Blyuss, Gordukova, Davydova, Zaikin, Zinovieva, Zimin, Molchanov, Salpagarova, Eremeeva, Filipenko, Prodeus, Korsunskiy, Hsu and Munblit. This is an open-access article distributed under the terms of the Creative Commons Attribution License (CC BY). The use, distribution or reproduction in other forums is permitted, provided the original author(s) and the copyright owner(s) are credited and that the original publication in this journal is cited, in accordance with accepted academic practice. No use, distribution or reproduction is permitted which does not comply with these terms. 